\title{
Hierarchical analysis of piecewise affine models of gene regulatory networks
}

\author{
Laurent Tournier and Jean-Luc Gouzé \\ INRIA COMORE, 2004 route des lucioles, 06902 Sophia-Antipolis, France \\ Laurent.Tournier@sophia.inria.fr, Jean-Luc.Gouze@sophia.inria.fr
}

\begin{abstract}
Summary. We propose in this paper a method to hierarchically organize a certain type of piecewise affine differential system. This specific class of dynamical systems has been extensively studied for the past few years, as it provides a good framework to model gene regulatory networks. Using the hierarchical organization of a piecewise affine system, we present a technique to qualitatively analyze the asymptotic behavior of the whole system thanks to the analysis of several smaller subsystems. Specifically adapted to these networks, an algorithm of threshold elimination is presented, that refines in certain cases the hierarchical decomposition and therefore improves the analysis.
\end{abstract}

\section{Introduction}

This article deals with hierarchical organization and hierarchical analysis of a class of piecewise affine systems of differential equations. This particular class of systems was first introduced by L. Glass in the 70's [10] as a model of genetic regulatory networks. Various aspects of these systems have been extensively studied since then, see e.g. [2, 6, 11], as they provide algorithmic methods to analyze qualitative dynamics of interaction networks. A computer tool, the Genetic Network Analyzer (GNA), has besides been elaborated to compute qualitative simulations of piecewise affine systems of relatively high dimensions [5].

Theoretically, a qualitative study of the dynamical behavior of a piecewise affine system consists in computing a transition graph, which can be seen as an abstraction of the phase portrait ${ }^{1}$. Such a study allows one to deduce asymptotic qualitative properties of the dynamics, such as the existence and the characterization of attractors. However, as the dimension of the system grows, finding these properties becomes harder. One way to improve this analysis is to take advantage of the structure of piecewise affine systems. The

\footnotetext{
${ }^{1}$ a directed edge exists between two regions if and only if there exists a solution of the system passing in finite time from the first to the second region.
} 
structure can be represented by an interaction graph, representing the mutual influences among the variables.

We propose here a method to decompose the interaction graph of a piecewise affine system in a hierarchical form [16]. This decomposition, based on the strongly connected components of the graph, has already been performed for general classes of dynamical systems [17]. The existing theorems are nevertheless quite general, and not really well-adapted to the piecewise affine framework. The paper is organized in three parts. We begin with some brief recalls about the piecewise affine framework. The second section deals with the hierarchical organization of the interaction graph, and with the asymptotic qualitative analysis of the dynamical behaviors of a piecewise affine system, based on the hierarchical decomposition. We briefly evoke in the last part an efficient pre-processing algorithm that may help this analysis by refining the decomposition. This algorithm has been successfully applied to a 9-dimensional system modeling the carbon starvation response network of $E$. coli bacterium, developped in [15].

\section{The framework of piecewise affine systems}

\subsection{Structure of a piecewise affine system}

We start with some headlines about the piecewise affine framework used to model gene regulatory networks. It will consist of some basic definitions and properties that we use in the rest of the paper; for a more exhaustive formulation of those systems, the reader is referred to $[5,6,11]$ and references therein. In the following, a "piecewise affine (PWA) system" designates a dynamical system of the form:

$$
\dot{x}(t)=K(x(t))-\Gamma(x(t)) x(t)
$$

where the state variable $x(t)$ is an $n$-dimensional vector of concentrations of different proteins produced by $n$ interacting genes. The production term $K(x)$ is a vector lying in $\mathbb{R}_{+}^{n}$ and the decay term $\Gamma(x)$ is an $n$-dimensional diagonal matrix $\Gamma(x)=\operatorname{diag}\left(\Gamma_{1}(x), \ldots, \Gamma_{n}(x)\right)$, with $\Gamma_{i}(x)>0$. System (1) can be written as $n$ ordinary differential equations:

$$
\dot{x}_{i}=K_{i}(x)-\Gamma_{i}(x) x_{i}, \quad x_{i} \geq 0
$$

For $i \in\{1, \ldots, n\}$, the state variable $x_{i}$ lies in a nonnegative interval $\left[0, \max _{i}\right]$. Therefore, the state vector $x(t)$ evolves within a hyperrectangular set $\Omega=$ $\prod_{i=1}^{n}\left[0, \max _{i}\right]$. To each $x_{i}$, we associate $p_{i}$ ordered positive constants: $0<$ $\theta_{i}^{1}<\theta_{i}^{2}<\cdots<\theta_{i}^{p_{i}}<\max _{i}$, called thresholds. By convention, for each $i \in\{1, \ldots, n\}$, we set $\theta_{i}^{0}=0$ and $\theta_{i}^{p_{i}+1}=\max _{i}$.

The production and degradation terms $K_{i}(x)$ and $\Gamma_{i}(x)$ share the same mathematical form. For $x \in \Omega$ and $i \in\{1, \ldots, n\}$ : 
Hierarchical analysis of piecewise affine systems

$$
K_{i}(x)=\sum_{l \in L_{i}} \kappa_{i}^{l} b_{i l}(x), \quad \Gamma_{i}(x)=\sum_{l \in L_{i}^{\prime}} \gamma_{i}^{l} b_{i l}^{\prime}(x)
$$

where $L_{i}$ and $L_{i}^{\prime}$ are (possibly empty) finite sets of indices, $\kappa_{i}^{l}$ and $\gamma_{i}^{l}$ are positive constants and the functions $b_{i l}$ and $b_{i l}^{\prime}$ are boolean valued. The functions $b_{i l}$ and $b_{i l}^{\prime}$ are often called regulation functions (see [6]) and are mathematically expressed by boolean expressions over the boolean variables $s^{+}\left(x_{i}, \theta_{i}^{j}\right)$, where $i \in\{1, \ldots, n\}, j \in\left\{1, \ldots, p_{i}\right\}$ and $s^{+}$is the so-called Heaviside (or step) function: $s^{+}(x, \theta)=0$ if $x<\theta$ and 1 if $x>\theta$ (we will denote $s^{-}$the function $\left.1-s^{+}\right)$.

The important consequence of these definitions is that a dynamical system defined by differential equations (2) and (3) is well-posed over the hyperrectangle $\Omega$ of $\mathbb{R}_{+}^{n}$, except on a subset of Lebesgue measure zero. Indeed, let us denote $H_{i, j}$ the hyperplane of $\mathbb{R}^{n}$ of equation $x_{i}=\theta_{i}^{j}$, for $i \in\{1, \ldots, n\}$ and $j \in\left\{0, \ldots, p_{i}+1\right\}$ and let $H$ be the subset: $H=\bigcup_{i=1}^{n}\left(\bigcup_{j=0}^{p_{i}+1} H_{i, j}\right)$.

The vector field $F(x)=K(x)-\Gamma(x) x$ is defined over $\Omega \backslash H$ (on each open hyperrectangle delimited by $H$, it is an affine vector field, with uncoupled equations) but it is discontinuous on the hypersurface $H$. This discontinuity of the vector field prevents us from defining global solutions in the classical sense, and forces us to consider, on the threshold hyperplanes, a special type of solutions known as Filippov solutions (see $[6,8]$ ).

With a slight abuse of language, we will call in the following a hyperrectangular domain of $\Omega$ an $n$-dimensional hyperrectangle included in $\Omega$, the faces of which are parallel to the axes and delimited in each direction $i$ by two hyperplanes $H_{i, j_{1}}$ and $H_{i, j_{2}}$, where $j_{1}<j_{2}$. Such a domain $\Delta$ is unequivocally characterized by a finite set of discrete equations of the form:

$$
\left\{\begin{array}{l}
s^{+}\left(x_{i}, \theta_{i}^{0}\right)=\cdots=s^{+}\left(x_{i}, \theta_{i}^{j_{1}}\right)=1 \\
s^{+}\left(x_{i}, \theta_{i}^{j_{2}}\right)=\cdots=s^{+}\left(x_{i}, \theta_{i}^{p_{i}}\right)=0
\end{array}\right.
$$

in each direction $i \in\{1, \ldots, n\}$. Particularly, and according to the literature on PWA systems, elementary domains defined by: $\left.D=\prod_{i=1}^{n}\right] \theta_{i}^{j_{i}}, \theta_{i}^{j_{i}+1}[$, for $j_{i} \in\left\{0, \ldots, p_{i}\right\}$ will be called regulatory domains or boxes.

Remark 1. To be complete and in accordance with the different articles about PWA systems, the regulatory domain $D$ defined above is a product of open intervals: $] \theta_{i}^{j_{i}}, \theta_{i}^{j_{i}+1}$ [ except if $j_{i}=0\left(\right.$ or $j_{i}=p_{i}$ ). In those cases, we will replace the open interval with the semi-open interval: $\left[0, \theta_{i}^{1}[\right.$ (respectively: $\left.] \theta_{i}^{p_{i}}, \max _{i}\right]$ ).

This definition of PWA system is quite heavy and an usual way to schematically represent such a system is to draw its interaction graph.

Definition 1 (Interaction graph). Consider an n-dimensional PWA system $\Sigma\left(n \in \mathbb{N}^{*}\right)$ The interaction graph of $\Sigma$ is the finite directed graph $\mathcal{G}_{\Sigma}=(\mathcal{V}, \mathcal{E})$ where $\mathcal{V}=\left\{v_{1}, \ldots, v_{n}\right\}$ (a vertex $v_{i} \in \mathcal{V}$ represents a gene), 
and the set of edges $\mathcal{E} \subset \mathcal{V} \times \mathcal{V}$ is defined as follows: for all couples of vertices $\left(v_{i}, v_{j}\right) \in \mathcal{V}^{2}$, there exists a directed edge from $v_{j}$ to $v_{i}\left(\right.$ noted $\left.\left(v_{j}, v_{i}\right) \in \mathcal{E}\right)$ if and only if $F_{i}(x)$ explicitly depends on $s^{+}\left(x_{j}, \theta_{j}^{k}\right)$ for some $k$.

Whenever $\left(v_{i}, v_{j}\right) \in \mathcal{E}$, we say that $x_{j}$ influences $x_{i}$ (or more rapidly that gene $j$ influences gene $i$ ). The interaction graph essentially captures the interactions between genes and therefore captures the structure of the system.

Remark 2. For a lot of examples in the literature, edges of the interaction graph are often labeled with a sign + or - , indicating whether the interaction is positive (activation or induction) or negative (repression or inhibition). Such a labeling is only possible for particular PWA systems. Indeed, for a general system, a gene $j$ may have both positive and negative actions on a gene $i$.

Example 1. Consider the three dimensional piecewise affine system given by:

$$
\begin{cases}\dot{x}_{1}=\kappa_{1}^{0} s^{+}\left(x_{2}, \theta_{2}^{1}\right) s^{+}\left(x_{3}, \theta_{3}^{1}\right)-\gamma_{1}^{0} x_{1}, & p_{1}=2 \\ \dot{x}_{2}=\kappa_{2}^{0}+\kappa_{2}^{1} s^{-}\left(x_{1}, \theta_{1}^{1}\right)+\kappa_{2}^{2} s^{+}\left(x_{2}, \theta_{2}^{1}\right)-\left(\gamma_{2}^{0}+\gamma_{2}^{1} s^{-}\left(x_{1}, \theta_{1}^{2}\right)\right) x_{2}, & p_{2}=1 \\ \dot{x}_{3}=\kappa_{3}^{0}+\kappa_{3}^{1} s^{+}\left(x_{1}, \theta_{1}^{1}\right)+\kappa_{3}^{2} s^{-}\left(x_{3}, \theta_{3}^{1}\right)-\left(\gamma_{3}^{0}+\gamma_{3}^{1} s^{-}\left(x_{1}, \theta_{1}^{2}\right)\right) x_{3}, & p_{3}=1\end{cases}
$$

The phase space of this system is the three dimensional hyperrectangle: $\Omega=\left[0, \max _{1}\right] \times\left[0, \max _{2}\right] \times\left[0, \max _{3}\right]$. The three dimensional phase space is composed of $3 \times 2 \times 2=12$ boxes. The interaction graph of $\Sigma$ is:

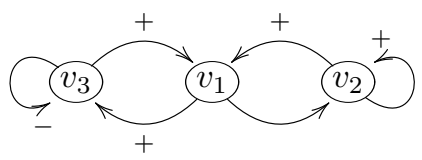

According to remark 2, this graph has been signed with respect to the signs of the interactions. The edge $\left(v_{1}, v_{2}\right)$ however could not be signed because $x_{1}$ has both positive and negative actions on $x_{2}$.

\subsection{Dynamics of a piecewise affine system}

In order to define properly the dynamics of system (1), we will have to partition $\Omega$ in different domains of the form: $D=D_{1} \times \cdots \times D_{n}$ where, for each $i \in\{1, \ldots, n\}, D_{i}$ is either a singleton $\left\{\theta_{i}^{j}\right\}$ for some $j \in\left\{1, \ldots, p_{i}-1\right\}$, or an interval $] \theta_{i}^{j}, \theta_{i}^{j+1}\left[\left(\right.\right.$ respectively $\left[0, \theta_{i}^{1}[\right.$ or $\left.\left.] \theta_{i}^{p_{i}}, \max _{i}\right]\right)$.

If, for all $i \in\{1, \ldots, n\}, D_{i}$ is not a singleton, then the domain $D$ is a regulatory domain, as we defined it in the previous section (it is also called regular). Otherwise, (i.e. if for at least one $i$ the set $D_{i}$ is reduced to a singleton), the domain $D$ is called a singular or switching domain (see [6]). We will respectively denote $\mathcal{D}_{r}$ and $\mathcal{D}_{s}$ the sets of regular and singular domains.

Over a regular domain $D \in \mathcal{D}_{r}$, the production and decay terms $K_{i}(x)$ and $\Gamma_{i}(x)$ are constants: $K_{i}(x)=\kappa_{i}^{D} \geq 0$, and $\Gamma_{i}(x)=\gamma_{i}^{D}>0$. System (1) is 
therefore a simple affine system in $D$, with $n$ uncoupled equations. It follows that $x(t)$ monotonically ${ }^{2}$ converges towards the point:

$$
\phi(D)=\left(\phi_{1}^{D}, \ldots, \phi_{n}^{D}\right)=\left(\frac{\kappa_{1}^{D}}{\gamma_{1}^{D}}, \ldots, \frac{\kappa_{n}^{D}}{\gamma_{n}^{D}}\right)
$$

which is an asymptotically stable equilibrium for the flow. This point is often called focal point of the domain $D$. In the literature about PWA systems, it is generally assumed that for any regular domain $D$, the focal point $\phi(D)$ belongs to $\Omega \backslash H$, i.e. $\phi(D)$ does not lie on a threshold hyperplane. Therefore $\phi(D)$ lies in a regular domain $D^{\prime} \in \mathcal{D}_{r}$. We have then to consider two cases, whether $D^{\prime}=D$ or not. If $D^{\prime}=D$ then the solution $x(t)$ belongs to $D$ for all $t \in \mathbb{R}_{+}$. So $\phi(D)$ is indeed an asymptotically stable equilibrium. In the other case, the trajectory escapes the domain $D$, i.e. there exists a finite time $t^{*}>0$ such that $x\left(t^{*}\right)$ belongs to a singular domain that bounds $D$. Sometimes it happens that the solution can be continued in the subsequent box without difficulties (see [2] and references therein). Otherwise, we have to consider singular dynamics over the singular domain.

Over a singular domain $D \in \mathcal{D}_{s}$, we already mentioned that the vector field is undefined. We thus cannot solve the differential equation in the classical sense. We can nevertheless use a weaker notion of solution which is known as Filippov solution (see [8]). We simply give here a brief summary of essential points, interested readers will find more details in PWA literature (see e.g. [2]).

Let $D \in \mathcal{D}_{s}$ be a singular domain. The Filippov method consists in extending the system (1) to a differential inclusion:

$$
\dot{x} \in H(x)=\overline{\mathrm{co}}\left(\left\{\kappa^{D^{\prime}}-\gamma^{D^{\prime}} x \mid D^{\prime} \in R(D)\right\}\right) \quad, \quad x(0)=x_{0} \in D
$$

where $R(D)=\left\{D^{\prime} \in \mathcal{D}_{r} \mid D \subseteq \partial D^{\prime}\right\}$ is the set of all regulatory domains which have $D$ in their boundary and $\overline{c o}(X)$ is the closed convex hull of $X$.

In the case where $H\left(x_{0}\right) \cap \operatorname{supp}(D)=\emptyset$, then the solution does not stay in $D$ and instantaneously escapes towards a regulatory domain. In the other case, we define a solution in the sense of Filippov as an absolutely continuous function $\xi(t)$ defined on $[0, T]$ such that $\xi(0)=x_{0}$ and $\dot{\xi}(t) \in H(\xi(t))$ for almost all $t \in[0, T]$. Such a solution exists for all initial conditions $x_{0}$ but is not guaranteed to be unique though, because of the generalization of the differential equation to a differential inclusion.

Several authors have studied the dynamics of PWA systems using Filippov method (see notably $[1,2,6,11]$ ). Following $[1,6]$, we will here use a slightly different definition of the differential inclusion (5):

$$
\dot{x} \in H(x)=\overline{\operatorname{rect}}\left(\left\{\kappa^{D^{\prime}}-\gamma^{D^{\prime}} x \mid D^{\prime} \in R(D)\right\}\right)
$$

\footnotetext{
${ }^{2}$ By this we mean that for each $i, x_{i}(t)$ is a monotone function of $t$.
} 
where $\overline{\operatorname{rect}}(X)$ designates the smallest closed hyperrectangle, the faces of which are parallel to the axes, containing the set $X$. This definition is clearly an over-approximation of (5) (see figure 1). Following the same authors, we

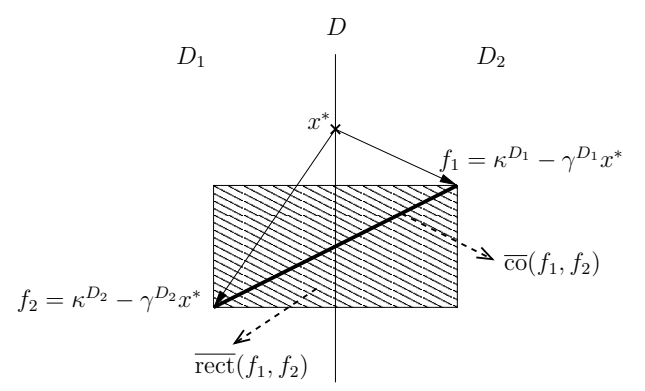

Fig. 1. Representations of $H(x)$ according to (5) and (6)

define, for a switching domain $D$ the notion of target equilibrium set ${ }^{3}$, which is a generalization of the focal points of regular domains:

Definition 2. Let $D \in \mathcal{D}_{s}$ be a switching domain. The target equilibrium set of $D$, noted $\Psi(D)$ is defined by: $\Psi(D)=\operatorname{supp}(D) \cap \overline{r e c t}\left(\left\{\phi\left(D^{\prime}\right) \mid D^{\prime} \in R(D)\right\}\right)$

The main interest of such sets lies in the following result (see [6]):

Lemma 1. Given a singular domain $D \in \mathcal{D}_{s}$ and an initial condition $x_{0} \in D$, any solution $\xi(t)$ of the differential inclusion (6) satisfies the property that for all $i \in\{1, \ldots, n\}, \xi_{i}(t)$ monotonically converges towards the orthogonal projection of $\Psi(D)$ on $\left[0, \max _{i}\right]: \pi_{i}(\Psi(D))=\left\{\psi_{i} \in\left[0, \max _{i}\right] \mid \psi \in \Psi(D)\right\}$

\section{Hierarchical organization of piecewise affine systems}

\subsection{The strongly connected components decomposition}

The notion of hierarchical organization of a PWA system relies on the well known strongly connected components (SCC) decomposition of the interaction graph. This work has already been done for more general dynamical systems (see e.g. [17]) according to a special definition of the interaction graph. For PWA systems, we will use the definition 1. According to this definition, the interaction graph of an $n$-dimensional PWA system $\Sigma$ is a digraph $\mathcal{G}_{\Sigma}=(\mathcal{V}, \mathcal{E})$ with $\operatorname{card}(\mathcal{V})=n$.

\footnotetext{
3 To be in accordance with PWA literature, we should have defined first focal sets as done for instance in [2]. We do not enter the details here, since definition 2 and lemma 1 will be sufficient for what follows.
} 
We recall here some basics about digraphs (see e.g. [3] for more details) Let $G=(V, E)$ be a digraph. Two vertices $u, v \in V$ are mutually reachable (denoted $u \sim v$ ) if and only if there exist two (directed) paths $\rho$ and $\rho^{\prime}$ such that $\rho$ joins $u$ to $v$ and $\rho^{\prime}$ joins $v$ to $u$. This relation is clearly an equivalence relation on the set $V$ of vertices. The strongly connected components of the digraph $G$ are then defined as the elements of $V / \sim$, that is to say the equivalence classes of the relation $\sim$. In other words, a strongly connected component of a digraph $G$ is a maximal set of vertices $C \subseteq V$ such that for every pair $u, v \in C, u$ and $v$ are reachable from each other.

The SCC decomposition of a digraph $G$ consists in computing the strongly connected components of $G: C_{1}, \ldots, C_{k}$ and then to compute the digraph $G^{s c c}=\left(V^{s c c}, E^{s c c}\right)$ defined as follows:

- $V^{s c c}=\left\{C_{1}, \ldots C_{k}\right\}$,

- given $i, j \in\{1, \ldots, k\}$ the edge $\left(C_{i}, C_{j}\right)$ belongs to $E^{s c c}$ if and only if there are $u \in C_{i}$ and $v \in C_{j}$ such that $(u, v) \in E$.

It can be easily proved (see [3]) that the digraph $G^{s c c}$ contains no (oriented) cycles. It is called a dag (for directed acyclic graph). This is a key property of $G^{s c c}$, because every dag can be topologically sorted (see [3], section 22.4). A topological sort of a dag can be viewed as a classification of its vertices in several hierarchical levels $H_{0}, H_{1}, \ldots$ such that the vertices of the first level $H_{0}$ are vertices with no predecessors, and the predecessors of vertices of level $H_{i}, i>0$ are contained in inferior levels $H_{j}$ with $j<i$ (see figure 2).
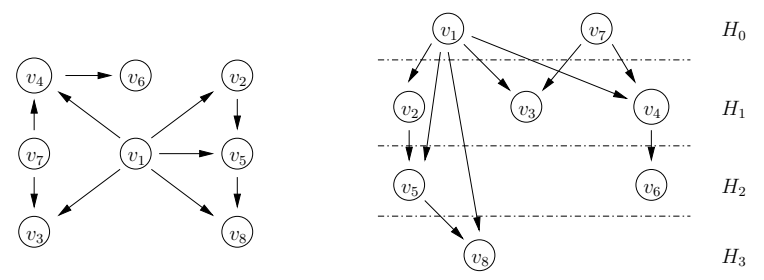

Fig. 2. A dag (on the left) and its topological sort (on the right), with 4 levels.

The interest of SCC decomposition of the interaction graph of a PWA system is quite obvious. Indeed, when performing this decomposition, we isolate several subsystems involving groups of variables that "work" together. These subsystems are ordered in several hierarchical levels which can allow the decomposition of the analysis of the whole system. Let us illustrate this by a simple 4-dimensional example.

Example 2. Consider the PWA system $(\Sigma)$ : 
Laurent Tournier and Jean-Luc Gouzé

$$
\left\{\begin{array}{l}
\dot{x}_{1}=\kappa_{1}^{0}+\kappa_{1}^{1} f_{1}(x)+\kappa_{1}^{2} s^{+}\left(x_{1}, \theta_{1}^{1}\right) s^{-}\left(x_{2}, \theta_{2}^{2}\right) s^{+}\left(x_{3}, \theta_{3}^{1}\right) f_{1}(x)-\gamma_{1} x_{1} \\
\dot{x}_{2}=\kappa_{2}^{0}+\kappa_{2}^{1} f_{2}(x)+\kappa_{2}^{2} s^{+}\left(x_{1}, \theta_{1}^{1}\right) s^{+}\left(x_{2}, \theta_{2}^{1}\right) f_{2}(x)-\gamma_{2} x_{2} \\
\dot{x}_{3}=\kappa_{3}^{0}+\kappa_{3}^{1} s^{-}\left(x_{4}, \theta_{4}^{1}\right)-\gamma_{3} x_{3} \\
\dot{x}_{4}=\kappa_{4}^{0}+\kappa_{4}^{1} s^{-}\left(x_{3}, \theta_{3}^{1}\right)-\gamma_{4} x_{4}
\end{array}\right.
$$

with $f_{1}(x)=\left(1-s^{-}\left(x_{1}, \theta_{1}^{2}\right) s^{+}\left(x_{2}, \theta_{2}^{1}\right)\right)$ and $f_{2}(x)=\left(1-s^{-}\left(x_{1}, \theta_{1}^{2}\right) s^{-}\left(x_{2}, \theta_{2}^{1}\right)\right)$. Its interaction graph and its SCC graph are represented by:

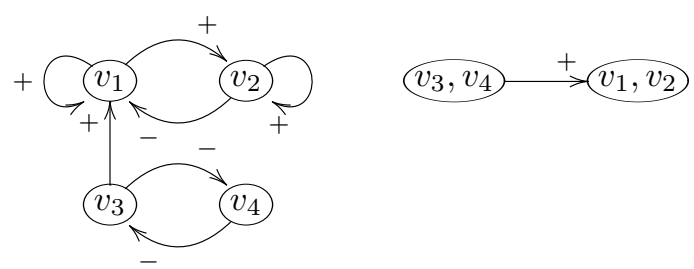

We have therefore decomposed system (7) into two isolated bidimensional subsystems with simple interaction graphs:

$\left(\Sigma_{1}\right)$

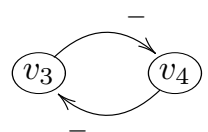

$\left(\Sigma_{2}\right)$

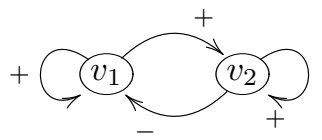

The first one $\left(\Sigma_{1}\right)$ is the well known "biological switch" often used in the literature to illustrate the concept of bistability (see [7] for theoretical aspects and [9] for experimental investigations). The second system is a negative loop with positive self-regulation of the two variables.

Under the following assumptions, giving the relative positions of focal points with respect to the thresholds:

$$
\left\{\begin{array}{l}
0<\kappa_{1}^{0} / \gamma_{1}<\theta_{1}^{1}<\left(\kappa_{1}^{0}+\kappa_{1}^{1}\right) / \gamma_{1}<\theta_{1}^{2}<\left(\kappa_{1}^{0}+\kappa_{1}^{1}+\kappa_{1}^{2}\right) / \gamma_{1}<\max _{1} \\
0<\kappa_{2}^{0} / \gamma_{2}<\theta_{2}^{1}<\left(\kappa_{2}^{0}+\kappa_{2}^{1}\right) / \gamma_{2}<\theta_{2}^{2}<\left(\kappa_{2}^{0}+\kappa_{2}^{1}+\kappa_{2}^{2}\right) / \gamma_{2}<\max _{2} \\
0<\kappa_{3}^{0} / \gamma_{3}<\theta_{3}^{1}<\left(\kappa_{3}^{0}+\kappa_{3}^{1}\right) / \gamma_{3}<\max _{3} \\
0<\kappa_{4}^{0} / \gamma_{4}<\theta_{4}^{1}<\left(\kappa_{4}^{0}+\kappa_{4}^{1}\right) / \gamma_{4}<\max _{4}
\end{array}\right.
$$

a qualitative analysis can be made of these simple bidimensional PWA systems. Figure 3 presents the two phase portraits. System $\left(\Sigma_{1}\right)$ shows two asymptotically stable steady states whereas $\left(\Sigma_{2}\right)$ shows an attractive cycle.

The method of qualitative simulation of PWA systems used in this example to compute the phase portraits of $\left(\Sigma_{1}\right)$ and $\left(\Sigma_{2}\right)$ is the one exposed in $[2$, $6]$. For such simple systems, this analysis can be carried out directly. We nevertheless recall the existence of the GNA software [5] that implements this technique and allows to qualitatively analyze higher dimensional PWA systems. The SCC decomposition has besides been implemented as a GNA module [4]. 

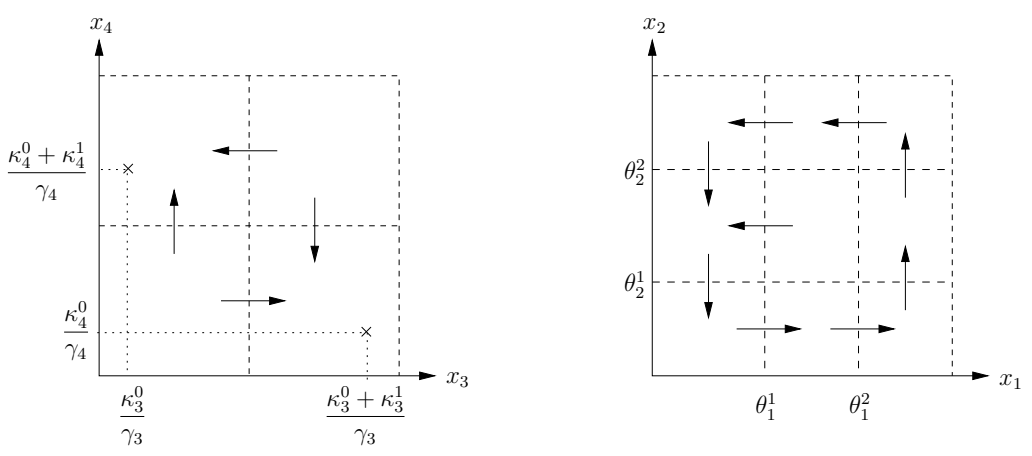

Fig. 3. Phase portraits of subsystems $\left(\Sigma_{1}\right)$ and $\left(\Sigma_{2}\right)$.

It must be noted that subsystems $\left(\Sigma_{1}\right)$ and $\left(\Sigma_{2}\right)$ are isolated, that is, unrelated among themselves. To rebuild the whole system $(\Sigma)$, one has to take into consideration the unique edge of the SCC graph which corresponds to the term $s^{+}\left(x_{3}, \theta_{3}^{1}\right)$ of the first equation of system (7). The question that is addressed in the next section is whether it is possible to deduce the qualitative behavior of the system (7) (or at least a part of its behavior) from the qualitative analysis of the isolated subsystems $\left(\Sigma_{1}\right)$ and $\left(\Sigma_{2}\right)$. This issue can be of particular importance if the initial system dimension is high because in that case a direct qualitative analysis of the whole system can lead to a huge transition graph.

\subsection{Asymptotic analysis of hierarchical PWA systems}

As we already said, the use of the SCC decomposition to analyze the behavior of a dynamical system is not new. In [17] for instance, Vidyasagar proposes to use such a decomposition for a more general class of dynamical systems, in order to express it in a triangular form:

$$
\dot{x}_{i}(t)=f_{i}\left(t, x_{1}(t), \ldots, x_{i}(t)\right), \quad i=1, \ldots, m
$$

Then, assuming that 0 is an equilibrium point for all isolated subsystems, he proves some powerful theorems (based on the general theory of dynamical systems) linking the stability of these equilibria and the stability of 0 as an equilibrium of the initial system. The underlying idea is actually quite simple: it consists in "injecting" the value of the equilibrium of the first subsystem into the second, then the value of the first and the second subsystems into the third, and so on until we obtain the whole system equilibrium.

Because of the discontinuities of the PWA vector fields, such general theorems are not directly applicable. We however propose in the following a technique specifically adapted to PWA framework that allows one to use the hierarchical organization of a PWA system for the analysis of its asymptotic 
dynamics. To present this method, we will use the system $(\Sigma)$ presented in example 2 as an illustrating example.

Let us therefore consider the PWA system (7) and let us assume the inequalities (8) on the parameters, placing the different focal points with respect to the thresholds. The letter $x$ designates the vector $\left(x_{1}, x_{2}, x_{3}, x_{4}\right)$ lying in $\left.\Omega=\prod_{i=1}^{4}\left[0, \max _{i}\right]\right)$. In the following, a hyperrectangular domain denotes an $n$-dimensional hyperrectangle, the faces of which are contained in threshold hyperplanes. We recall that such a domain is unequivocally denoted by a finite set of discrete equations. These domains will take an essential part because according to the definition of a PWA system (see equation (3)), the interactions between variables take place only by means of qualitative values, i.e. by means of terms of the form $s^{+}\left(x_{i}, \theta_{i}^{j}\right)$. Therefore, instead of "injecting" the exact value of an equilibrium into a subsequent system, we will only have to inject its qualitative value, that is, a set of discrete equations involving the discrete variables $s^{+}\left(x_{i}, \theta_{i}^{j}\right)$.

Let us first focus on the subsystem $\left(\Sigma_{1}\right)$. As we said before, this system shows two attractors (see figure 3 ), which are the two regular steady states:

$$
A=\left(\left(\kappa_{3}^{0}+\kappa_{3}^{1}\right) / \gamma_{3}, \kappa_{4}^{0} / \gamma_{4}\right) \text { and } A^{\prime}=\left(\kappa_{3}^{0} / \gamma_{3},\left(\kappa_{4}^{0}+\kappa_{4}^{1}\right) / \gamma_{4}\right)
$$

Remark 3. There is actually a third equilibrium point which is the singular steady state $\left(x_{3}, x_{4}\right)=\left(\theta_{3}^{1}, \theta_{4}^{1}\right)$. This steady state will be neglicted here as it can be easily shown to be unstable (see [2] for a precise definition of stability and unstability of singular steady states).

Let $\Delta$ and $\Delta^{\prime}$ be the smallest hyperrectangular domains containing respectively the two steady states: $\left.\Delta=] \theta_{3}^{1}, \max _{3}\right] \times\left[0, \theta_{4}^{1}\left[\right.\right.$ and $\Delta^{\prime}=\left[0, \theta_{3}^{1}[\times] \theta_{4}^{1}, \max _{4}\right]$. These sets are given by their discrete equations:

$$
\Delta:\left\{\begin{array}{l}
s^{+}\left(x_{3}, \theta_{3}^{1}\right)=1 \\
s^{+}\left(x_{4}, \theta_{4}^{1}\right)=0
\end{array} \quad \text { and } \quad \Delta^{\prime}:\left\{\begin{array}{l}
s^{+}\left(x_{3}, \theta_{3}^{1}\right)=0 \\
s^{+}\left(x_{4}, \theta_{4}^{1}\right)=1
\end{array}\right.\right.
$$

System $\left(\Sigma_{1}\right)$ has therefore two types of trajectories: the first ones converging towards the attractor $A$ and the second ones towards $A^{\prime}$ (we neglect here the trajectories leading to $\left(\theta_{3}^{1}, \theta_{4}^{1}\right)$, see remark 3$)$. According to which attractor we are heading to, we respectively inject the discrete equations of $\Delta$ or $\Delta^{\prime}$ into the system $\left(\Sigma_{2}\right)$. We consequently have to consider two cases:

- If $\left(x_{3}, x_{4}\right)$ converges towards $A$, then $s^{+}\left(x_{3}, \theta_{3}^{1}\right)=1$ and the bidimensional system involving the variables $x_{1}$ and $x_{2}$ can be replaced by:

$$
\left(\Sigma_{2}\right)\left\{\begin{array}{l}
\dot{x}_{1}=\kappa_{1}^{0}+\kappa_{1}^{1} f_{1}(x)+\kappa_{1}^{2} s^{+}\left(x_{1}, \theta_{1}^{1}\right) s^{-}\left(x_{2}, \theta_{2}^{2}\right) f_{1}(x)-\gamma_{1} x_{1} \\
\dot{x}_{2}=\kappa_{2}^{0}+\kappa_{2}^{1} f_{2}(x)+\kappa_{2}^{2} s^{+}\left(x_{1}, \theta_{1}^{1}\right) s^{+}\left(x_{2}, \theta_{2}^{1}\right) f_{2}(x)-\gamma_{2} x_{2}
\end{array}\right.
$$

- If, on the contrary, $\left(x_{3}, x_{4}\right)$ converges towards $A^{\prime}$, then $s^{+}\left(x_{3}, \theta_{3}^{1}\right)=0$ and thus we replace $\left(\Sigma_{2}\right)$ by: 


$$
\left(\Sigma_{2}^{\prime}\right)\left\{\begin{array}{l}
\dot{x}_{1}=\kappa_{1}^{0}+\kappa_{1}^{1} f_{1}(x)-\gamma_{1} x_{1} \\
\dot{x}_{2}=\kappa_{2}^{0}+\kappa_{2}^{1} f_{2}(x)+\kappa_{2}^{2} s^{+}\left(x_{1}, \theta_{1}^{1}\right) s^{+}\left(x_{2}, \theta_{2}^{1}\right) f_{2}(x)-\gamma_{2} x_{2}
\end{array}\right.
$$

We have removed the dependency in $x_{3}$ and therefore have reduced the analysis of the 4-dimensional system (7) to the analysis of two uncoupled bidimensional systems.

Before carrying on and completing the analysis of this special system, we have to justify that such a simplification is correct. The proof that follows concerns the current example, however it is pretty straightforward and can easily be generalized to any similar situation.

It relies on two main facts. First, $\Delta$ and $\Delta^{\prime}$ are positively invariants ${ }^{4}$ for the flow of subsystem $\left(\Sigma_{1}\right)$ (this comes directly from the fact that $A$ and $A^{\prime}$ are regular steady states of $\left.\left(\Sigma_{1}\right)\right)$. The second fact is that these sets are reached in finite time, given (almost) any initial condition $\left(x_{3}^{0}, x_{4}^{0}\right) \in\left[0, \max _{3}\right] \times\left[0, \max _{4}\right]$. This result is quite obvious and directly comes from the qualitative analysis of the dynamics of system $\left(\Sigma_{1}\right)$ (see fig. 3). For a better understanding of what happens, we make here an additional assumption: $\gamma_{3}=\gamma_{4}=\gamma$. With this assumption, the trajectories in each box can easily be shown to be straight segments, and it becomes possible to draw the basins of attraction $\mathcal{B}(A)$ and $\mathcal{B}\left(A^{\prime}\right)$ of the two attractors (see figure 4 ). It must be noted that the points

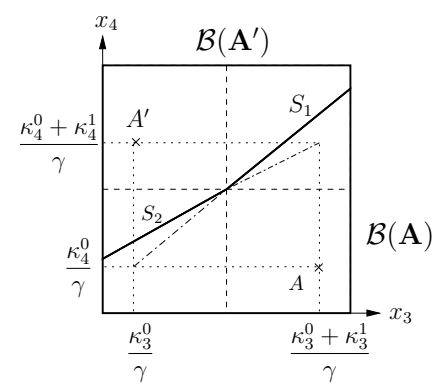

Fig. 4. Basins of attraction of the two attractors $A$ and $A^{\prime}$.

lying in the boundary between $\mathcal{B}(A)$ and $\mathcal{B}\left(A^{\prime}\right)$ (which is composed of the union of the two segments $S_{1} \cup S_{2}$, see figure 4) will be supposed not to belong to these basins, as they all lead to the unstable steady state $\left(\theta_{3}^{1}, \theta_{4}^{1}\right)$. We are now able to formalize the previous statement:

Proposition 1. Let $x^{0} \in \Omega$ such that $\left(x_{3}^{0}, x_{4}^{0}\right) \notin S_{1} \cup S_{2}$, and let $x(t)$ be the solution of (7) with $x(0)=x^{0}$. Then, whatever $x_{1}^{0}$ and $x_{2}^{0}$, there exists a finite time $t^{*} \in \mathbb{R}_{+}$such that $\left(x_{3}\left(t^{*}\right), x_{4}\left(t^{*}\right)\right) \in \Delta$ (respectively $\Delta^{\prime}$ ) and $\Xi=$ $\left\{\left(x_{1}(t), x_{2}(t)\right) \mid t \geq t^{*}\right\}$ is the solution of system $\left(\Sigma_{2}\right)$ (resp. $\left.\left(\Sigma_{2}^{\prime}\right)\right)$, starting from the point $\left(x_{1}\left(t^{*}\right), x_{2}\left(t^{*}\right)\right)$ at time $t^{*}$.

\footnotetext{
${ }^{4}$ This is a classical notion in dynamical systems theory, see e.g. [13].
} 
According to this proposition, and after analyzing the phase portraits of systems $\left(\Sigma_{2}\right)$ and $\left(\Sigma_{2}^{\prime}\right)$, we can complete the asymptotic qualitative analysis of system (7). We actually find two main attractors, which are a regular steady state contained in the box: $] \theta_{1}^{1}, \theta_{1}^{2}\left[\times\left[0, \theta_{2}^{1}\left[\times\left[0, \theta_{3}^{1}[\times] \theta_{4}^{1}, \max _{4}\right]\right.\right.\right.$ and an attractive cycle contained in the region: $\left.\left.\left[0, \max _{1}\right] \times\left[0, \max _{2}\right] \times\right] \theta_{3}^{1}, \max _{3}\right] \times\left[0, \theta_{4}^{1}[\right.$.

\subsection{Generalization for other types of attractors}

We have so far restricted ourselves to a very particular kind of attractors which are regular steady states. It must be noted that this is not a limitation for the method and other types of attractors can be considered as well (see for instance figure 5).

Consider, for $n \in \mathbb{N}^{*}$, an $n$-dimensional PWA system given by equations (2) and (3). Suppose that its SCC decomposition leads to the hierarchical graph:

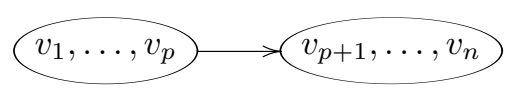

Let $y$ and $z$ denote the subvectors:

$$
\left\{\begin{array}{l}
y=\left(y_{1}, \ldots, y_{p}\right)=\left(x_{1}, \ldots, x_{p}\right) \\
z=\left(z_{1}, \ldots, z_{n-p}\right)=\left(x_{p+1}, \ldots, x_{n}\right)
\end{array}\right.
$$

and suppose that the $y$-subsystem has a unique global attractor $\mathcal{A} \subset \Omega_{y}=$ $\prod_{i=1}^{p}\left[0, \max _{i}\right]$. In order to apply the previous technique, we will have to define a hyperrectangle hull of $\mathcal{A}$ :

Definition 3. Let $p \in \mathbb{N}^{*}$ and $\Omega=\prod_{i=1}^{p}\left[0, \max _{i}\right]$. Given a subset $\Lambda$ of $\Omega$, we call hyperrectangular hull of $\Lambda$ (noted $\Delta(\Lambda)$ ), the smallest p-dimensional hyperrectangle, the faces of which are contained in threshold hyperplanes, that contains $\Lambda$. The set $\Delta(\Lambda)$ is unequivocally determined by a finite set of discrete equations.

Following the technique presented in the previous section, we state the following proposition:

Proposition 2. If we suppose that the $y$-subsystem has a unique global attractor $\mathcal{A} \subset \Omega_{y}$, then the asymptotic behavior of the $z$-subsystem is the same as the asymptotic behavior of the z-system in which we have injected the discrete equations of the hyperrectangle $\Delta(\mathcal{A})$.

Although we do not give the proof here, it mainly relies on the two key points precedently evoked: the set $\Delta(\mathcal{A})$ is positively invariant for the subsystem in $y$, and given any initial condition $x^{0} \in \Omega$, there exists a finite time $t^{*} \in \mathbb{R}_{+}$ such that $y\left(t^{*}\right) \in \Delta(\mathcal{A})$. Obviously, if the system in $y$ shows several attractors, then a complete analysis leads to consider subsequently the different cases. 
Contrary to the case of regular steady states, where the hyperrectangular hull is reduced to the box containing the steady state, for other attractors, the hull can contain many boxes, and can even be equal to the whole phase space. In those cases, the technique may fail to uncouple the two subsystems. This is actually to relate to a general issue for all model reduction techniques, which is the issue of irreducibility of systems.

Another limitation of the presented approach is that its effectiveness is directly related to the SCC decomposition. Obviously it is not applicable if, for instance, the interaction graph of the initial system is already strongly connected. it may also happen for real biological high dimensional systems, that the SCC decomposition has a main strongly connected component of relatively high dimension, which is not very satisfactory from a model reduction point of view. For this reason, we propose in the next part a simple algorithm that may be able to solve this problem, for certain systems, by "cutting" this main component into smaller ones. This technique has been successfully applied to the example of the carbon starvation in E. coli bacterium [15].

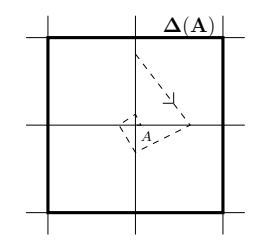

A singular steady state

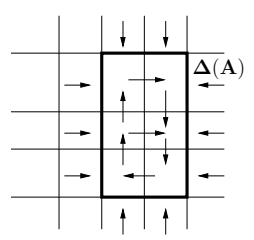

A more complex attractor

Fig. 5. Two types of attractors (2-d) with their hyperrectangular hulls.

\section{Threshold elimination, application to a biological model}

We propose in this section a simple and straightforward preprocessing treatment whose goal is to possibly refine the model reduction of the SCC decomposition. It is based on the simple following observation. Let us consider a $n$-dimensional PWA system $(\Sigma)$. We compute, for each regulatory domain $D \in \mathcal{D}_{r}$, the focal point $\phi(D)$ with formula (4). We denote by $\Phi$ the set of all focal points: $\Phi=\left\{\phi(D) \mid D \in \mathcal{D}_{r}\right\}$.

Lemma 2. If, for $i \in\{1, \ldots, n\}$, there exist $j_{1}, j_{2} \in\left\{1, \ldots, p_{i}\right\}$ such that for all $\phi \in \Phi, \theta_{i}^{j_{1}}<\phi_{i}<\theta_{i}^{j_{2}}$, then, let $\left.\Delta^{(i)}=\left[0, \max _{1}\right] \times \ldots\right] \theta_{i}^{j_{1}}, \theta_{i}^{j_{2}}\left[\ldots\left[0, \max _{n}\right]\right.$ :

(i) $\Delta^{(i)}$ is positively invariant for $(\Sigma)$.

(ii) Given any initial condition $x^{0} \in \Omega, \exists t^{*} \in \mathbb{R}_{+}, x\left(t^{*}\right) \in \Delta^{(i)}$. 
This can be easily deduced from lemma 1 , as, for all domains $D$ (both regular and singular) the attractive equilibrium set $\Psi(D)$ is contained in the set $\Delta^{(i)}$.

This provides us with a simple procedure to eliminate the thresholds $\theta_{i}^{1}, \ldots, \theta_{i}^{j_{1}}$ and $\theta_{i}^{j_{2}}, \ldots, \theta_{i}^{p_{i}}$ (by replacing $s^{+}\left(x_{i}, \theta_{i}^{0}\right), \ldots, s^{+}\left(x_{i}, \theta_{i}^{j_{1}}\right)$ by 1 and $s^{+}\left(x_{i}, \theta_{i}^{j_{2}}\right), \ldots, s^{+}\left(x_{i}, \theta_{i}^{p_{i}}\right)$ by 0$)$. By doing this, we possibly cut some edges of the interaction graph, and therefore possibly refine the SCC decomposition. The two major advantages of this procedure are that it can be performed directly on the structure of the PWA system regardless of its dynamics, and it can also be performed independently in each direction $i \in\{1, \ldots, n\}$.

The method presented in this paper, together with the threshold elimination algorithm have been succesfully applied (see [16] for details) to a real biological example: the carbon starvation response network in the bacterium E. coli. The piecewise affine model of this network can be found in two different versions $[14,15]$. In both cases, the SCC decomposition has a relatively big component, which is not satisfactory. After threshold elimination, we managed to refine it with a central three dimensional component (the same for the two versions), a complete dynamical analysis of which can be found in [12].

\section{Conclusion}

The method presented in this article provides an algorithmic way to analyze a PWA dynamical system of relatively high dimension, analyzing several smaller dynamical systems. From a mathematical point of view, this is to be related with the general concept of model reduction. The concept of model reduction of a dynamical system is not a well-posed mathematical problem. Indeed, according to the expression of the differential equations of the system, there are many ways to reduce a system. The goal, however, is always the same: it consists in simplifying the system in order to make its dynamical study easier. In the present paper, the term "reduction" must be understood in a very specific way. It corresponds to the reduction of the number of vertices and edges of the interaction graph. As we saw in the 4-dimensional example in the second part, cutting a particular edge can lead to uncouple two subsystems of the whole system, and therefore drastically simplify the analysis. We must be aware though that this situation is specific and for higher dimensional systems such simplifications will certainly rarely happen. It is mainly for this reason that we proposed the threshold elimination algorithm in the last part. Indeed, it can be easily implemented and allows, as a preprocessing treatment, a rapid simplification of a system. The application on the real example of carbon starvation response seems quite encouraging. So as to show its practical relevance, the method presented here has to be tested on other examples of real biological models. 


\section{References}

1. G. Batt, H. de Jong, J. Geiselmann, M. Page, D. Ropers, and D. Schneider. Symbolic reachability analysis of genetic regulatory networks using qualitative abstraction. Technical Report RR-5362, INRIA, 2004.

2. R. Casey, H. de Jong, and J.-L. Gouzé. Piecewise-linear models of genetic regulatory networks: equilibria and their stability. J. Math. Biol., 52:27-56, 2006.

3. T.H. Cormen, C.E. Leiserson, R. L. Rivest, and C. Stein. Introduction to algorithms. MIT Press, second edition, 2001.

4. D.A. Cristescu. Algorithmic study on genetic regulatory networks. Technical report, Automatic control and computer science faculty, Politechnica University of Bucharest, 2006. internship report, INRIA.

5. H. de Jong, J. Geiselmann, C. Hernandez, and M. Page. Genetic network analyser: qualitative simulation of genetic regulatory networks. Bioinformatics, 19(3):336-344, 2003.

6. H. de Jong, J.-L. Gouzé, C. Hernandez, M. Page, T. Sari, and J. Geiselmann. Qualitative simulation of genetic regulatory networks using piecewise-linear models. Bull. Math. Biol., 66:301-340, 2005.

7. E. Farcot and J.-L. Gouzé. How to control a biological switch: a mathematical framework for the control of piecewise affine models of gene networks. Technical Report RR-5979, INRIA, 2006. url: https://hal.inria.fr/inria-00094853.

8. A.F. Filippov. Differential equations with discontinuous righthand sides. Kluwer Academic Publishers, Boston, 1988.

9. T.S. Gardner, C.R. Cantor, and J.J. Collins. Construction of a genetic toggle switch in Escherichia coli. Nature, 403:339-342, 2000.

10. L. Glass. Combinatorial and topological methods in nonlinear chemical kinetics. J. Chem. Phys., 63:1325-1335, 1975.

11. J.-L. Gouzé and T. Sari. A class of piecewise linear differential equations arising in biological networks. Dyn. Syst., 17(4):299-316, 2002.

12. F. Grognard, J.-L. Gouzé, and H. de Jong. Piecewise-linear models of genetic regulatory networks: theory and example. In Biology and control theory: current challenges, Lecture Notes in Control and Information Sciences (LNCIS). Springer-Verlag. (to appear).

13. L. Perko. Differential equations and dynamical systems, volume 7 of Texts in Applied Mathematics. Springer Verlag, 1991.

14. D. Ropers. An extended model of the carbon starvation response in Escherichia coli. In preparation.

15. D. Ropers, H. de Jong, M. Page, D. Schneider, and J. Geiselmann. Qualitative simulation of the carbon starvation response in Escherichia coli. Biosystems, 84(2):124-152, 2006.

16. L. Tournier and J.-L. Gouzé. Hierarchical analysis of piecewise affine models of gene regulatory networks. Technical Report RR-6189, INRIA, 2007. url: https://hal.inria.fr/inria-00145357.

17. M. Vidyasagar. Decomposition techniques for large-scale systems with nonadditive interactions: stability and stabilizability. IEEE Transactions on Automatic Control, (4):773-779, 1980. 